\title{
Homozygous stop-gain variant in $L R R C 32$, encoding a TGF $\beta$ receptor, associated with cleft palate, proliferative retinopathy, and developmental delay
}

\author{
Tamar Harel $\mathbb{D}^{1} \cdot$ Ephrat Levy-Lahad ${ }^{2} \cdot$ Muhannad Daana $^{3} \cdot$ Hadas Mechoulam $^{4} \cdot$ Smadar Horowitz-Cederboim $^{2}$. \\ Michal Gur ${ }^{1} \cdot$ Vardiella Meiner $^{1} \cdot$ Orly Elpeleg $^{1,5}$
}

Received: 19 June 2018 / Revised: 1 March 2019 / Accepted: 7 March 2019 / Published online: 11 April 2019

(c) European Society of Human Genetics 2019

\begin{abstract}
The transforming growth factor-beta (TGF $\beta$ ) signaling pathway is essential for palatogenesis and retinal development. Glycoprotein A repetitions predominant (GARP), encoded by $L R R C 32$, is a TGF $\beta$ cell surface receptor that has been studied primarily in the context of cellular immunity. We identified a homozygous stop-gain variant in LRRC32 (c.1630C $>\mathrm{T}$; p.(Arg544Ter)) in two families with developmental delay, cleft palate, and proliferative retinopathy. Garp-null mice have palate defects and die within $24 \mathrm{~h}$ after birth. Our study establishes LRRC32 as a candidate disease-associated gene in humans and lends further support to the role of the TGF $\beta$ pathway in palatogenesis and retinal development.
\end{abstract}

\section{Introduction}

The transforming growth factor-beta (TGF $\beta$ ) superfamily is a crucial regulatory cytokine family with pleiotropic functions in immunity, cancer, and development, including neurogenesis, palatogenesis, and retinal development. Sequential waves of activation, inhibition, and reactivation of TGF $\beta$ family members help orchestrate development of the nervous system, from early embryogenesis through

Supplementary information The online version of this article (https:// doi.org/10.1038/s41431-019-0380-y) contains supplementary material, which is available to authorized users.

$\triangle$ Tamar Harel

tamarhe@hadassah.org.il

1 Department of Genetic and Metabolic Diseases, Hadassah-Hebrew University Medical Center, 9112001 Jerusalem, Israel

2 Medical Genetics Institute, Shaare Zedek Medical Center, Faculty of Medicine, Hebrew University of Jerusalem, Jerusalem, Israel

3 Child Development Centers, Clalit and Maccabi Health Care Services, Jerusalem District, Jerusalem, Israel

4 Center for Pediatric Ophthalmology, Department of Ophthalmology, Hadassah-Hebrew University Medical Center, Jerusalem, Israel

5 Monique and Jacques Roboh Department of Genetic Research, Hadassah-Hebrew University Medical Center, Jerusalem, Israel adulthood. TGF $\beta$ members regulate development of dopaminergic neurons in the midbrain, proliferation of cerebellar neurons, neural migration, and axon guidance. Additionally, TGF $\beta$ members seem to have important roles in neuron repair following brain injury and in neurodegenerative disease [1].

LRRC32 encodes glycoprotein A repetitions predominant (GARP), a cell surface receptor for TGF $\beta$. GARP has been extensively studied as a cell surface non-signaling receptor on regulatory T-lymphocytes, platelets, and certain cancer cells. It is responsible for cell-surface docking of latent TGF $\beta$ before activation and release of the mature cytokine, and has an important role in maintaining peripheral tolerance and preventing inflammatory diseases [2-4]. Knockdown of GARP in regulatory $T$ cells dramatically decreased their immune suppressive capacity [5], while increased expression of GARP has been suggested to promote cancer progression through activation of immunosuppressive functions of regulatory T cells [6]. Despite vast investigations, LRRC32 has not yet been associated with a monogenic Mendelian disease.

Recently, Garp knockout mice were observed to die within $24 \mathrm{~h}$ after birth, with defective palatogenesis yet no other major organ abnormalities. Decreased apoptosis and SMAD2 phosphorylation at the medial edge of epithelial cells of the palatal shelves at E14.5 dpc indicated a defect in the TGF $\beta$ signaling pathway, and in vitro studies confirmed that GARP and TGF $\beta 3$ directly interact. GARP was 
shown to be indispensable for surface expression and activation of membrane-associated latent TGF $\beta 3$ during embryogenesis [7].

In this study, we report a homozygous stop-gain variant in LRRC32 in three affected individuals from two families who presented with developmental delay, cleft palate, and proliferative retinopathy. We present the clinical and molecular features of the syndrome in light of current knowledge regarding TGF $\beta$ signaling in midfacial and retinal morphogenesis.

\section{Materials and methods}

\section{Exome analysis}

Following informed consent, exome analysis was pursued on DNA extracted from whole blood of individual III-3 of Family A and individual III-1 of Family B (Fig. 1a). Exonic sequences from DNA were enriched with the SureSelect Human All Exon $50 \mathrm{Mb}$ V4 Kit (Agilent Technologies, Santa Clara, California, USA). Sequences were generated
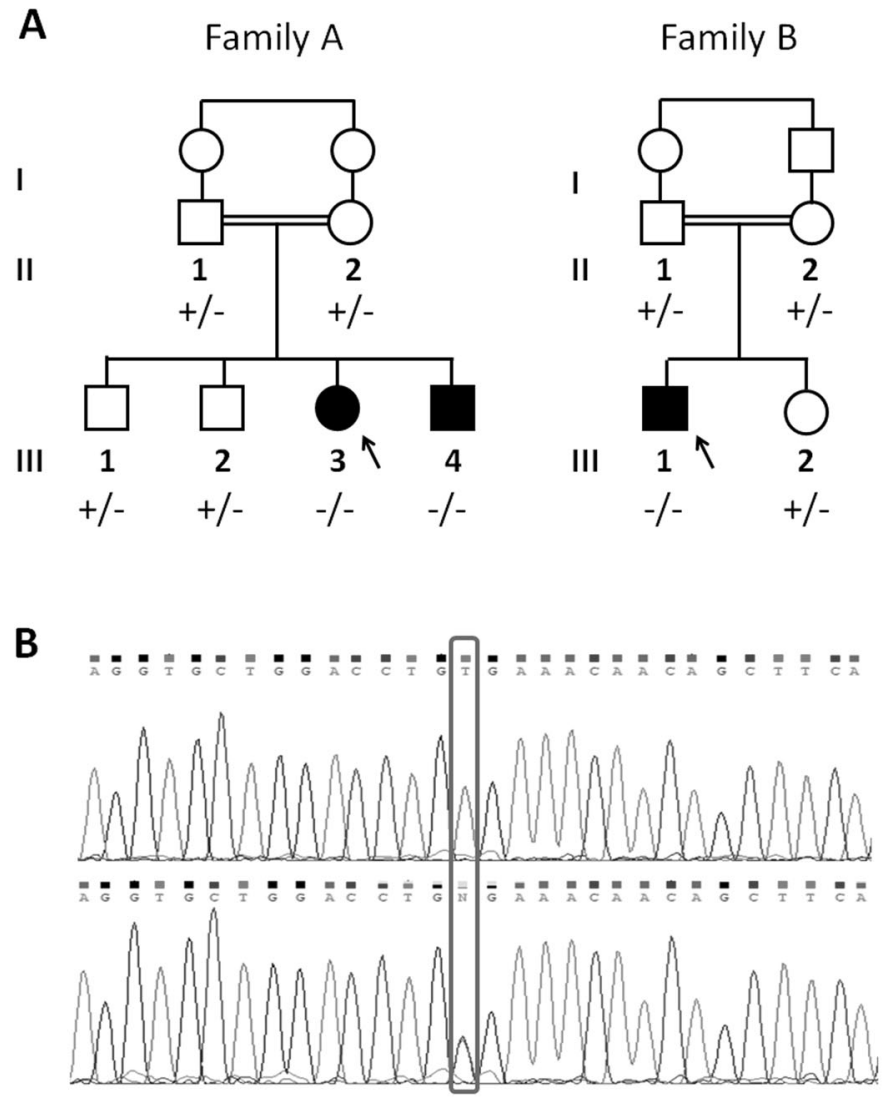

Fig. 1 Pedigree, molecular analysis, and brain magnetic resonance imaging (MRI) images of affected individuals. a Pedigrees of both families, indicating segregation of the LRRC32 stopgain variant. Note the parental consanguinity in both pedigrees. b Sanger traces of on a HiSeq2500 (Illumina, San Diego, California, USA) as 125-bp paired-end runs. Read alignment and variant calling were performed with DNAnexus (Palo Alto, California, USA) using default parameters with the human genome assembly hg19 (GRCh37) as reference. Exome analysis of the probands yielded 52.9 million and 35.4 million mapped reads, with a mean coverage of $96 \times$ and $61 \times$ (individuals III-3 of Family A and III-1 of Family B, respectively).

\section{Segregation analysis}

Amplicons containing the LRRC32 variant were amplified by conventional PCR of genomic DNA, and analyzed by Sanger dideoxy nucleotide sequencing.

\section{Results}

\section{Clinical reports}

Detailed clinical evaluations are available in the Supplementary Material and in Table 1. Individuals III-3 and III-4

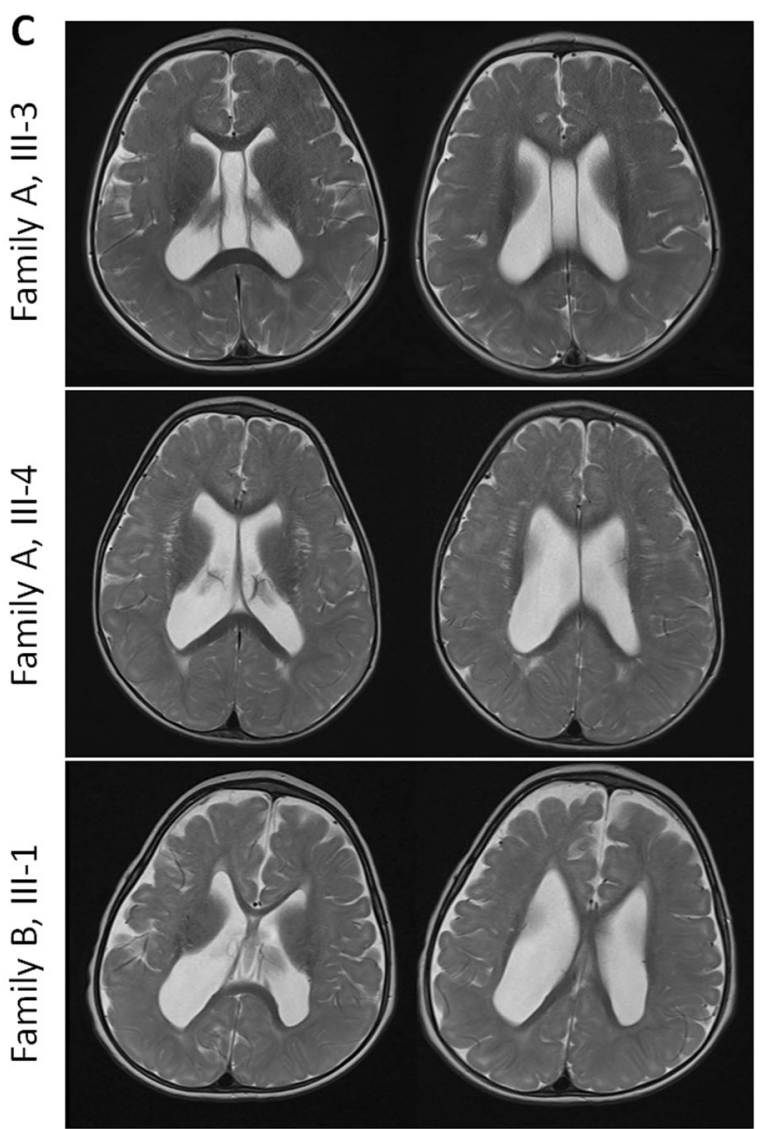

individuals with homozygous (upper panel) and heterozygous (lower panel) $L R R C 32$ c. $1630 \mathrm{C}>\mathrm{T}$ variants. c Axial T2-weighted brain MRI images of affected individuals, demonstrating slightly asymmetrical, enlarged lateral ventricles 
Table 1 Clinical features of affected individuals

\begin{tabular}{|c|c|c|c|}
\hline & $\begin{array}{l}\text { Family A, Individual } \\
\text { III-3 }\end{array}$ & $\begin{array}{l}\text { Family A, Individual } \\
\text { III-4 }\end{array}$ & $\begin{array}{l}\text { Family B, Individual } \\
\text { III-1 }\end{array}$ \\
\hline Age at last exam & 3 years -2 months & 2 years -11 months & 3 years -3 months \\
\hline Gender & $\mathrm{F}$ & M & M \\
\hline Gestational age & 40 weeks +3 days & 34 weeks & 36 weeks \\
\hline Birth weight ( $Z$ score) & $2380 \mathrm{~g}(-2.56)$ & $1340(-2.2)$ & $1740 \mathrm{~g}(-2.81)$ \\
\hline $\begin{array}{l}\text { Global developmental } \\
\text { delay }\end{array}$ & + & + & + \\
\hline DQ & 72 & 57 & 23 \\
\hline Age at walking & 24 months & 27 months & Non-ambulatory \\
\hline Speech delay & + & + & + \\
\hline Height ( $Z$-score) & $88.5 \mathrm{~cm}(-1.74)$ & $86 \mathrm{~cm}(-2.39)$ & $90 \mathrm{~cm}(-1.89)$ \\
\hline Weight (Z- score) & $13.2 \mathrm{~kg}(-0.65)$ & $12.5 \mathrm{~kg}(-1.23)$ & $12 \mathrm{~kg}(-2.03)$ \\
\hline $\begin{array}{l}\text { Head circumference } \\
\text { (Z-score) }\end{array}$ & $48.2 \mathrm{~cm}(-0.3)$ & $47 \mathrm{~cm}(-1.6)$ & $46.8(-1.7)$ \\
\hline Dysmorphic features & Midface retrusion & Micrognathia & Short philtrum \\
\hline Hypotonia & + & + & $\begin{array}{l}+(\text { axial hypotonia, } \\
\text { increased peripheral tone })\end{array}$ \\
\hline Atopic dermatitis & + & + & NA \\
\hline Cleft palate & + & + & + \\
\hline Myopia & + & + & + \\
\hline Proliferative retinopathy & + & + & + \\
\hline Strabismus & - & + & - \\
\hline Hearing loss & Mild to moderate & Mild to moderate & - \\
\hline Brain MRI & $\begin{array}{l}\text { Cavum septum } \\
\text { pellucidum, mild } \\
\text { ventriculomegaly }\end{array}$ & Ventriculomegaly & $\begin{array}{l}\text { Ventriculomegaly, partial } \\
\text { agenesis corpus } \\
\text { callosum, vermian } \\
\text { hypoplasia }\end{array}$ \\
\hline Echocardiography & NA & Normal & Normal \\
\hline Sleep apnea & + & NA & + \\
\hline
\end{tabular}

$D Q$ developmental quotient, $F$ - female, $M$ male, $M R I$ magnetic resonance imaging, $N A$ not available from Family A (Fig. 1a) were siblings born to first cousins of Palestinian descent, and manifested developmental delay, cleft palate, proliferative retinopathy, and mild to moderate hearing loss. Developmental quotient (DQ) varied from borderline low in the female $(D Q=72$ at 3 years) to severely delayed in the male $(D Q=57)$. Physical examination revealed hypotonia, a clumsy gait, and atopic dermatitis in addition to the repaired cleft palate. Ophthalmological findings included high myopia, pale optic discs and bilateral proliferative retinopathy for Individual III-3 in Family A (Fig. 2), and esotropia, optic atrophy, and proliferative retinopathy in Individual III-4 in Family A. Electroretinogram/visual-evoked potential was available for the younger child, and revealed evidence of widespread retinal disease. Brain magnetic resonance imaging (MRI) showed ventriculomegaly (Fig. 1c). Chromosomal microarray (CMA) did not reveal any known diseaseassociated variation, and exome sequencing was pursued.

The proband in Family B was a male, the first of two children born to first cousin parents of Palestinian descent.

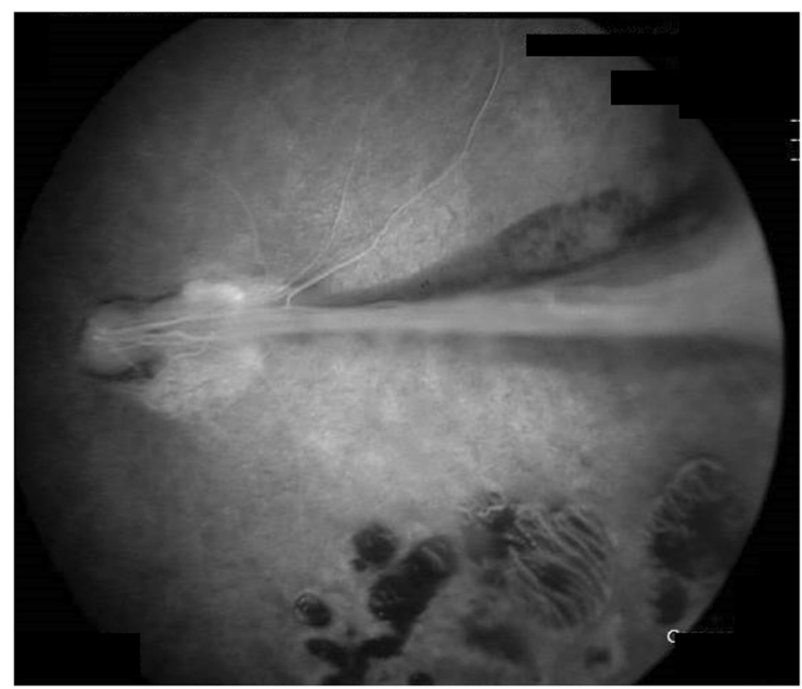

Fig. 2 Fluorescein imaging of fundus. Fluorescein imaging of the left fundus of Family A, individual III-3, showing a large retinal fold from the optic disc temporally and peripheral laser marks 
He had marked developmental delay, cleft palate, blindness, cataract, and bilateral retinal detachment. Development was more severely delayed as compared to Family A. At age 3 years, he was nonverbal and could not crawl. He had severe obstructive sleep apnea requiring nighttime noninvasive ventilation. Physical exam revealed axial hypotonia with increased peripheral tone and upgoing Babinski. MRI demonstrated ventriculomegaly, widened subarachnoid spaces, partial agenesis of the corpus callosum, hypoplastic cerebellar vermis, and Dandy Walker malformation. Extensive evaluations (see Supplementary material) including CMA were nonrevealing, and exome sequencing was therefore pursued.

\section{Exome sequencing identifies a homozygous stop- gain variant in LRRC32}

To identify an underlying genetic diagnosis, exome sequencing was pursued on the proband in both families. Following alignment to the reference genome [hg19] and variant calling, variants were filtered out if the total read depth was less than $8 \times$, and if they were off-target ( $>8 \mathrm{bp}$ from splice junction), synonymous, or had minor allele frequency $(\mathrm{MAF})>0.001$ in the in-house and GnomAD databases. The affected individuals shared two homozygous variants, one in LRRC32 (chr11:g.76371007G >A [hg19]; NM_005512.2: c.1630C $>$ T, p.(Arg544Ter)) and the other in TENM4 (chr11:g.78565274C >T [hg19]; NM_001098816.2; c. $1556 \mathrm{G}>\mathrm{A}$, p.(Gly519Glu)), on a shared haplotype of 10.4 Mb (chr11: 74431552-84822798). Ascertainment of the connection between the families was done by a genotype-first approach, whereby the homozygous LRRC32 stop-gain variant in one proband prompted an internal database search for additional cases with variants in the same gene. Although the families were not aware of any blood relation between them, the shared haplotype indicated common ancestry, suggesting a founder mutation. The LRRC32 variant leads to a premature termination codon (PTC) at position 544 of 662 amino acids. Since the PTC lies in the last exon, the mRNA transcript is predicted to escape nonsense mediated decay [8], and the protein would be missing the transmembrane domain (TMD) as well as a short cytoplasmic domain [9]. The identified variant was previously seen once in GnomAD, in the heterozygous state. It segregated with the disease in available family members (Fig. 1a). Notably, within our exome database comprising 3500 individuals, with $\sim 50 \%$ Palestinians, no other carriers were identified. TENM4, which harbored the second shared variant, is associated with autosomal dominant essential tremor. Although this does not exclude its involvement in autosomal recessive disease, the clinical manifestations of retinopathy and palatogenesis are more readily explained by the LRRC32 homozygous stop-gain variant.

\section{Discussion}

In this study, we report two families with cleft palate, proliferative retinopathy, and developmental delay (Table 1), in whom genetic analysis revealed a shared homozygous stop-gain variant in LRRC32 (c.1630C $>\mathrm{T}$, p. (Arg544Ter)). The more significant developmental delay of the proband in the second family could either reflect the severe end of the phenotypic spectrum of this newly defined disease, or may be explained by a dual diagnosis of LRRC32 and a second gene. While no additional rare variants were identified in known disease-associated genes that could explain the severe developmental delay, we cannot rule out the contribution of a second novel diseaseassociated gene (Table S1).

LRRC32 encodes GARP, which consists of 662 amino acids that form a long extracellular domain with 20 leucinerich repeats (LRR) ending at residue 628, a single hydrophobic TMD and a short carboxy-terminal cytoplasmic tail [9]. Thus, even if the p.(Arg544Ter) transcript is stable, it would fail to produce the TMD and the intracellular domain. GARP protein shares $\sim 80 \%$ similarity with the murine Garp, which is expressed during late organogenesis of the mouse. At day 14.5 dpc, Garp mRNA was detected in regions of skin invagination; in cephalic structures such as the eye, nasal cavity, and choroid plexus; and in muscle [10].

Garp knockout mice exhibit neonatal lethality and defective palatogenesis via defective TGF $\beta$ signaling [7]. Similarly, mice lacking TGF $\beta 3$ have defective palatogenesis, presumably secondary to abnormal epithelialmesenchymal interaction [11-13]. Moreover, TGF $\beta$ signaling regulates IRF6 in the palate through SMAD4 [14], and rare variants in IRF6 are associated with Van der Woude syndrome, an autosomal dominant ectodermal dysplasia that includes orofacial clefting and lip pits [15]. Thus, the human phenotype of cleft palate associated with a homozygous stop-gain variant in LRRC32 partially resembles that of the knockout mice.

Interestingly, Garp knockout mice were not noted to have retinal defects; however, Tgfb2(-/-) Tgfb3(-/-) double-deficient mice have been shown to display severe alterations in the morphology of the retina, lens, and cornea, including retinal detachment [16]. Moreover, TGF $\beta$ signaling has been shown to mediate programmed cell death in the developing chick retina, crucial to proper retinal development [17]. During retinal development, TGF $\beta$ members have been shown to control programmed cell death and cell density in chick and mouse models [17-19]. TGF $\beta$ signaling is also required for maintenance of retinal ganglion cell differentiation and survival [20]. Patients with proliferative vitreoretinal diseases exhibit overexpression of TGF $\beta$ in the vitreous, and downstream mediators of TGF $\beta$ have been highlighted as therapeutic targets [21]. 
GARP has been studied in the context of atopic dermatitis [22]. Of note, the affected individuals studied herein had no indication of immune deficiency, yet those in Family A did suffer from atopic dermatitis. Future studies of additional individuals with LRRC32 variants will confirm or refute this association.

In conclusion, our data suggest that $L R R C 32$ as a candidate disease gene associated with a clinical presentation characterized by developmental delay, cleft palate, and proliferative retinopathy. This study highlights the utility of careful phenotyping of mouse knockout models in anticipation of human data. Further studies in both humans and animal models are required to substantiate causation and to evaluate the specific role of LRRC32/GARP in neurogenesis and in ocular development and maintenance.

\section{Accession Numbers}

The ClinVar accession numbers for the DNA variant data reported in this paper are SCV000778511, SCV000886489, SCV000886519, SCV000886520, and SCV000886521.

Acknowledgements The authors wish to thank the families for their participation in this study as well as Dr. Shimon Edvardson for help with MRI images and Dr. Ela Shai for technical assistance.

\section{Compliance with ethical standards}

Conflict of interest The authors declare that they have no conflict of interest.

Publisher's note: Springer Nature remains neutral with regard to jurisdictional claims in published maps and institutional affiliations.

\section{References}

1. Meyers EA, Kessler JA. TGF- $\beta$ family signaling in neural and neuronal differentiation, development, and function. Cold Spring Harb Perspect Biol. 2017;9:a022244.

2. Metelli A, Salem M, Wallace CH, Wu BX, Li A, Li X, et al. Immunoregulatory functions and the therapeutic implications of GARP-TGF- $\beta$ in inflammation and cancer. J Hematol Oncol. 2018;11:24.

3. Rachidi S, Metelli A, Riesenberg B, Wu BX, Nelson MH, Wallace $\mathrm{C}$, et al. Platelets subvert $\mathrm{T}$ cell immunity against cancer via GARP-TGF $\beta$ axis. Sci Immunol. 2017;2:eaai7911.

4. Wallace CH, Wu BX, Salem M, Ansa-Addo EA, Metelli A, Sun $\mathrm{S}$, et al. B lymphocytes confer immune tolerance via cell surface GARP-TGF- $\beta$ complex. JCI Insight 2018;3;e99863.

5. Fridrich S, Hahn SA, Linzmaier M, Felten M, Zwarg J, Lennerz $\mathrm{V}$, et al. How soluble GARP enhances TGF $\beta$ activation. PLoS ONE. 2016;11:e0153290.
6. Miyazono K, Katsuno Y, Koinuma D, Ehata S, Morikawa M. Intracellular and extracellular TGF- $\beta$ signaling in cancer: some recent topics. Front Med. 2018;12:387-411.

7. Wu BX, Li A, Lei L, Kaneko S, Wallace C, Li X, et al. Glycoprotein A repetitions predominant (GARP) positively regulates transforming growth factor (TGF) $\beta 3$ and is essential for mouse palatogenesis. J Biol Chem. 2017;292:18091-7.

8. Khajavi M, Inoue K, Lupski JR. Nonsense-mediated mRNA decay modulates clinical outcome of genetic disease. Eur J Hum Genet. 2006;14:1074-81.

9. Ollendorff V, Noguchi T, deLapeyriere O, Birnbaum D. The GARP gene encodes a new member of the family of leucine-rich repeat-containing proteins. Cell Growth Differ. 1994;5:213-9.

10. Roubin R, Pizette S, Ollendorff V, Planche J, Birnbaum D, Delapeyriere O. Structure and developmental expression of mouse Garp, a gene encoding a new leucine-rich repeat-containing protein. Int J Dev Biol. 1996;40:545-55.

11. Kaartinen V, Voncken JW, Shuler C, Warburton D, Bu D, Heisterkamp N, et al. Abnormal lung development and cleft palate in mice lacking TGF-beta 3 indicates defects of epithelialmesenchymal interaction. Nat Genet. 1995;11:415-21.

12. Proetzel G, Pawlowski SA, Wiles MV, Yin M, Boivin GP, Howles PN, et al. Transforming growth factor-beta 3 is required for secondary palate fusion. Nat Genet. 1995;11:409-14.

13. Taya Y, O'Kane S, Ferguson MW. Pathogenesis of cleft palate in TGF-beta3 knockout mice. Development. 1999;126:3869-79.

14. Iwata J, Suzuki A, Pelikan RC, Ho TV, Sanchez-Lara PA, Urata $M$, et al. Smad4-Irf6 genetic interaction and TGF $\beta$-mediated IRF6 signaling cascade are crucial for palatal fusion in mice. Development. 2013;140:1220-30.

15. Kondo S, Schutte BC, Richardson RJ, Bjork BC, Knight AS, Watanabe Y, et al. Mutations in IRF6 cause Van der Woude and popliteal pterygium syndromes. Nat Genet. 2002;32:285-9.

16. Dünker N, Krieglstein K. Reduced programmed cell death in the retina and defects in lens and cornea of Tgfbeta2(-/-) Tgfbeta3(-/-) double-deficient mice. Cell Tissue Res. 2003;313:1-10.

17. Dünker N, Schuster N, Krieglstein K. TGF-beta modulates programmed cell death in the retina of the developing chick embryo. Development. 2001;128:1933-42.

18. Murali D, Kawaguchi-Niida M, Deng CX, Furuta Y. Smad4 is required predominantly in the developmental processes dependent on the BMP branch of the TGF- $\beta$ signaling system in the embryonic mouse retina. Invest Ophthalmol Vis Sci. 2011;52: 2930-7.

19. Duenker N. Transforming growth factor-beta (TGF-beta) and programmed cell death in the vertebrate retina. Int Rev Cytol. 2005;245:17-43.

20. Walshe TE, Leach LL, D'Amore PA. TGF- $\beta$ signaling is required for maintenance of retinal ganglion cell differentiation and survival. Neuroscience. 2011;189:123-31.

21. Kita T, Hata Y, Arita R, Kawahara S, Miura M, Nakao S, et al. Role of TGF-beta in proliferative vitreoretinal diseases and ROCK as a therapeutic target. Proc Natl Acad Sci USA. 2008; 105:17504-9.

22. Manz J, Rodríguez E, ElSharawy A, Oesau EM, Petersen BS, Baurecht $\mathrm{H}$, et al. Targeted resequencing and functional testing identifies low-frequency missense variants in the gene encoding GARP as significant contributors to atopic dermatitis risk. J Invest Dermatol. 2016;136:2380-6. 